\title{
Aprendiendo los cuidados del hogar en contextos migratorios: ambivalencias, alianzas y distancias*
}

\author{
Learning Home Care in Migratory Contexts: \\ Ambivalences, Alliances and Distances
}

Recibido: 31 de octubre de 2014 | Aceptado: 14 de agosto de 2015

\author{
LAURA CRISTINA YUFRA ${ }^{* *}$ \\ CONICET-Universidad Nacional de Jujuy (UNJU) \\ y As. ERAPI-Laboratorio Cooperativo de Socioantropología. \\ ENRIQUE SANTAMARÍA LORENZO*** \\ As. ERAPI-Laboratorio Cooperativo de Socioantropología y Universidad de Barcelona (UB)
}

doi :10.11144/Javeriana.upsy14-5.achc

Para citar este artículo: Yufra, L., \& Santamaría, E. (2015). Aprendiendo los cuidados del hogar en contextos migratorios: ambivalencias, alianzas y distancias. Universitas Psychologica, 14(5), 1637-1648. http://dx.doi.org/10.11144/Javeriana. upsy14-5.achc

* Artículo de investigación. Con el apoyo del Comisionado para las Universidades y la Investigación del Departamento de Innovación, Universidades y Empresas del Gobierno de Cataluña y del Fondo Social Europeo.

** Dra. en Psicología Social. Correo electrónico: laurayufra@yahoo.com.ar

*** Dr. en Sociología. E-mail:esantamaria@ub.edu
RESUMEN

El artículo busca problematizar las dinámicas que se establecen entre usuarias y formadoras de un curso de cuidados del hogar, dirigido a personas inmigrantes en situación irregular en la ciudad de Barcelona. A partir del análisis de dichas dinámicas, perfilamos la ambivalencia de los dispositivos formativos implementados para ofrecer ayuda o apoyo a las personas inmigrantes. En dichos dispositivos es posible reconocer posiciones sociales jerarquizadas y distantes —nacional/inmigrante-extranjero-, así como alianzas en las identificaciones comunes — la "empleada"/la "señora"-. A la luz del análisis, se hace patente, primero, la necesidad de las tareas de cuidado para la reproducción de la vida y, segundo, su escaso reconocimiento social y su exigua remuneración y cobertura legal — cuando son ofrecidas en el mercado laboral-.

Palabras clave

inmigración; formación para la inserción laboral; cuidados; clasificaciones sociales; Barcelona

\section{A B S T R A C T}

This paper seeks to debate the dynamics existing between users of and trainers in a home care course for illegal immigrants in Barcelona. The analysis of these dynamics reveals the ambivalence of the help and support devices used by immigrants. These devices expose distant and top-down social positions (Spanish citizen / immigrant-foreigner), as well as alliances between users and trainers based on their shared identification as "employees" visà-vis the "lady of the house". Our analysis also reveals the importance of care work for the reproduction of life and also the lack of social recognition, low pay and poor legal coverage this type of job gets in the labour market. Keywords

immigration; workforce training; care; social classifications; Barcelona 


\section{Introducción}

El artículo busca analizar las dinámicas que tienen lugar en un curso de formación para la inserción laboral dirigido a personas inmigrantes que se encuentran en una situación administrativa irregular en la ciudad de Barcelona. Los resultados emergen del trabajo de campo realizado para la elaboración de una tesis doctoral ${ }^{1}$. Asimismo, es preciso señalar que el proceso investigativo se vio enriquecido por la participación de Laura Yufra en proyectos de investigación que abordaron la articulación entre movilidades humanas, género y recursos de atención dirigidos a mujeres inmigrantes ${ }^{2}$. También fueron de suma importancia los debates que se desarrollaron en el marco de un grupo de trabajo que los autores de este texto coordinan. ${ }^{3}$

En las últimas décadas, hemos presenciado un acercamiento entre las perspectivas de género y el tratamiento de las migraciones. La feminización de las migraciones es el resultado de intersecciones específicas, más concretamente, de las relaciones transnacionales que buscan satisfacer las demandas de trabajo productivo y reproductivo, de las políticas migratorias y familiares (Anderson, 2000; Sassen, 2003; Gutiérrez Rodríguez, 2013) y de las estrategias que las y los migrantes utilizan para 'superar' las trabas que enfrentan los movimientos poblacionales (Pedone, 2002). En el caso español, la feminización de las migraciones se explica, entre otras cosas, por

1 La mencionada tesis tuvo por título "Inmigrantes en formación: Estado de bienestar y promoción de lazos sociales en el caso de la inserción laboral de las personas inmigrantes en Barcelona" y se defendió públicamente en el Departamento de Psicología Social de la Universidad Autónoma de Barcelona en diciembre de 2012.

2 Los proyectos de investigación mencionados son: "Mirando hacia la igualdad. Pertinencia sociocultural de los servicios sociales para mujeres inmigradas ( U-18/06)" financiado por el Instituto Catalán de las mujeres y "Mujeres inmigrantes y sistema educativo en la sociedad receptora: Análisis y propuestas de mejoramiento del acceso y la satisfacción en los recursos de formación para la inserción sociolaboral (088/07)" financiado por el Instituto de la mujer. El grupo de que las llevó a cabo es el grupo "Fractalidades en Investigación Crítica" de la Universidad Autónoma de Barcelona integrado por Marisela Montenegro, Caterine Galaz, Karla Montenegro y Laura Yufra.

3 Nos referimos al grupo de Trabajo del Instituto Catalán de Antropología ERAPI-Socioantropología de los mundos contemporáneos del cual surge la Asociación Erapi - Laboratorio Cooperativo de Sociantropología. la demanda de cuidados generada por la entrada de las mujeres españolas al mercado laboral, por la no distribución de las tareas de cuidado entre los géneros (Parella Rubio, 2000) y por el escaso desarrollo del Estado de Bienestar español, que se caracteriza por ser "familista" (Moreno, 2002; Ferrera, 1996). Son las familias —en particular las mujeres - las que se encargan de impartir bienestar a sus miembros (Gil Araujo \& González-Fernández, 2014). Estos elementos indican que la demanda de cuidados es satisfecha, fundamentalmente, por mujeres inmigrantes y en situaciones muy precarias y socialmente poco valoradas.

Durante el periodo en el que se realizó esta investigación (2009-2011), se intensificó, en Cataluña, la aplicación de las lógicas neoliberales en los servicios de atención a la población, produciendo recortes presupuestarios y transformaciones en los modos de organización de las políticas públicas, con una particular incidencia en el financiamiento de los programas de integración social de las personas inmigrantes. Esto se hizo evidente, entre otras cosas, con la disminución de los recursos económicos destinados al Fondo de Apoyo a la Acogida e Integración de Inmigrantes y al Refuerzo. En el 2009, su presupuesto fue de doscientos millones de euros; durante el 2010, de setenta millones y, en el 2011, de sesenta y seis millones de euros (Foro para la integración social de los inmigrantes, 2011, p. 193).

Otro ejemplo de la tendencia mencionada es la negación del acceso a la sanidad para las personas inmigrantes en situación irregular. Aunque el ahorro monetario que dicha exclusión generaría no ha sido comprobado, sí ha colocado a las personas inmigrantes en una situación de mayor vulnerabilidad. Además, ha fortalecido los efectos simbólicos de ilegitimidad de la aspiración de dichas personas al derecho a la salud e, incluso, de su propia presencia en el territorio nacional-estatal, vuelve vulnerables a las personas inmigrantes y fortalece los efectos simbólicos de su exclusión, en términos de la ilegitimidad de su aspiración al derecho a la salud y en relación con su propia presencia. Por otra parte, las políticas sociales relativas al cuidado entre ellas la Ley de Dependencia del 2006-, que apostaron por hacer una transferencia monetaria a 
las familias en lugar de desarrollar servicios sociales públicos, vieron disminuidos sus recursos financieros a causa de las políticas de austeridad, adoptadas para atajar la crisis económica (Gil Araujo \& González-Fernández, 2014).

El análisis del curso de formación en cuidados del hogar nos permitió reflexionar sobre los modos en que las mujeres inmigrantes acceden a algunos recursos del sistema de bienestar — en este caso, el acceso a un proceso de formación-. Reflexionamos sobre los contenidos que se imparten en el curso, sobre lo que se entiende por "cuidado del hogar" y sobre los modos de llevarlo a cabo. Asimismo reconocemos, en las relaciones sociales establecidas en el curso, tanto sobre lo que se entiende por "cuidado del hogar" como sobre los modos de llevarlo a cabo. Cómo se (re)producen y desestabilizan ciertas clasificaciones sociales, identificando la dinámica en la que se generan dichas clasificaciones y su articulación específica (Brah, 2004). Con este ejercicio pretendemos explicar cómo acaecen las discriminaciones de género, de origen nacional y de posición social, poniendo de manifiesto por qué las mujeres inmigrantes las que están principalmente "destinadas" a llevar a cabo tareas de cuidado, poco valoradas y con escasa retribución monetaria en un contexto de crisis económica y en un país semiperiférico del sur de Europa. El interés por la formación para la inserción laboral de las personas inmigrantes proviene de que este resulta sumamente revelador, en términos de los mecanismos de clasificación social presentes en las que aquellas se instalan, así como de los contenidos que las tareas del cuidado tienen y de las características de las personas "idóneas" para llevarlas a cabo.

\section{Método y materiales}

Este trabajo se centra en el análisis de algunas situaciones densamente significativas, a las que accedimos a partir de la observación participante y de las entrevistas informales que fueron efectuadas durante el curso de inserción laboral mencionado anteriormente. El curso fue dictado en una iglesia de la ciudad de Barcelona y auspiciado por una entidad católica. Las notas periodísticas y los documentos elaborados por las administraciones públicas, relativos a la formación para la inserción laboral de las personas inmigrantes, como parte de las políticas de integración de las mismas, constituyeron materiales secundarios.

La selección del curso se realizó a partir de un cruce de criterios: por su aparición reiterada en varios motores de internet; por su inclusión en el folleto informativo "Barcelona ciudad de trabajo" entregado en las sesiones orientadoras de la entidad Barcelona Activa, dependiente del Gobierno de Cataluña y del Ayuntamiento de Barcelona, y de su señalamiento como sitio de referencia para personas inmigrantes por parte de algunos informantes clave. Es importante decir que la realización del curso es un prerrequisito para obtener una certificación emitida por la entidad y para acceder a la bolsa de trabajo gestionada por la coordinadora de formación. Si bien el curso está dirigido a mujeres, pudimos constatar que asisten también algunos hombres ${ }^{4}$.

Las observaciones se realizaron durante diciembre del 2009 y enero del 2010, y asistimos a todas las clases que conformaban el curso. Entre julio del 2009 y finales de mayo del 2011, se llevaron a cabo tareas de preparación y análisis del trabajo de campo. Durante el desarrollo del mencionado curso, pudimos entrevistar de manera informal a todos los participantes - formadoras y asistentes-, principalmente en los momentos de pausa, de bienvenida o de despedida de las clases.

La aproximación metodológica por la que optamos se deriva de una perspectiva de investigación de corte cualitativo que aspira a la comprensión contextual de las prácticas sociales. Las observa-

4 Las fechas de realización del trabajo se corresponden con la crisis económica desatada en el 2008, que afectó particularmente a los modos de inserción típicos de los hombres migrantes — como la construcción-. Las tareas del cuidado se revelan como un trabajo mal remunerado, pero menos sujeto a los vaivenes del mercado, debido a la necesidad intensiva de mano de obra. La aceptación de hombres en el curso fue justificada por la demanda de algunas tareas de cuidado que requieren mucha fuerza física, como la movilización de personas con graves incapacidades físicas, como nos lo informó la coordinadora de la formación. Por otra parte, podríamos hablar de la feminización de ciertos trabajos que implican la informalidad, la indeterminación entre el horario de trabajo y el no trabajo, la capacidad de gestionar simultáneamente múltiples tareas y habilidades de tipo afectivo relacional, entre otras (Vargas Monroy \& Pujal, 2014). 
ciones in situ permitieron elaborar una descripción detallada de las prácticas cotidianas que se producen en los cursos de inserción laboral (Hammersley $\&$ Atkinson, 1994). En esta forma particular de producir conocimiento, los resultados de la investigación están mediados por las estrategias metodológicas empleadas (Velasco \& Díaz de Rada, 1997; Taylor \& Bodgan, 1986). No obstante, según esta perspectiva, el afán por conocer una situación singular también ofrece luces acerca del funcionamiento de los mundos sociales que conforman tal situación (Berteaux, 2005). En este sentido, el análisis del espacio social en el que se desarrolla el curso de formación nos permitió reconocer cómo funcionan algunas categorías relativas a la inmigración - en particular, en lo que respecta a la pertenencia y la exclusión-y cómo se proyectan, sobre esta, valores y actitudes de los mundos sociales que le atañen, como los mercados laborales, la sociedad mayoritaria, etc. Las preguntas que hicimos, de manera informal y abierta, buscaron que las personas interpeladas, explicaran su punto de vista sobre sí mismas y sobre las demás personas que como ellas, se encontraban implicadas een la realización de la formación en cuidados del hogar. Así, pudimos comprender las lógicas prácticas (Bourdieu, 2008) puestas en juego en las relaciones sociales que se producían durante el desarrollo del curso. Las estrategias de coproducción de la información estuvieron orientadas al reconocimiento de categorías a través de las cuales algunos sujetos son construidos como carentes y necesitados de intervención (Montenegro, 2001; Montenegro, Montenegro, Yufra, \& Galaz, 2011) y de políticas específicas (Shore \& Wrigth, 1997).

En el marco de esta aproximación metodológica, la posición de la investigadora es entendida como una vía de acceso al conocimiento. Cabe señalar que, en el proceso de trabajo de campo, se ocuparon diferentes posiciones sociales: usuaria inmigrante de los cursos $-\mathrm{y}$, en tanto tal, aprendiza de los cuidados del hogar- e investigadora universitaria no implicada directamente en la relación de aprendizaje, entre otras. Estas posiciones no siempre respondieron a una decisión de la investigadora, sino al lugar que los y las participantes le asignaron a ella. Por lo anterior, las posiciones sociales ocupadas ofrecen perspectivas de análisis diferenciales.

\section{Resultados}

La asignación de recursos y la implementación de estrategias socioasistenciales dirigidas a personas inmigrantes tiene como fin brindar apoyo y facilitar el establecimiento de la población recién llegada. En este sentido, las políticas y las prácticas que se dirigen a tal población buscan subsanar la situación de desventaja en la que esta se encuentra, dada su condición de extranjería (a la que puede añadirse la irregularidad administrativa) y su situación socioeconómica. Sin embargo, como veremos a continuación, no siempre se logran tales objetivos - declarados explícitamente en las políticas dirigidas a la población inmigrante-. En lo que sigue y a partir de los resultados de nuestra investigación, veremos las distintas formas de acceso al curso de formación en cuestión, las relaciones sociales que allí se establecen, los contenidos de la formación, las personas que se construyen como "ideales" para llevar a cabo ciertas tareas y las características que estas personas deben tener. A la luz de estos resultados, señalaremos la construcción de desigualdades y dinámicas excluyentes, en los mandatos de género, en las distinciones en relación con el origen nacional y en las posiciones sociales ocupadas.

\section{Cursos para personas inmigrantes: acceso condicionado.}

Las administraciones públicas en Cataluña han asumido un criterio normalizador para el tratamiento de la inmigración. Esto es, que los inmigrantes deben ser atendidos en los equipamientos, pogramas y servicios dirigidos al conjunto de la población (Sanahuja i Velez (2010). En la práctica existen excepciones a dicho principio normalizador. Es el caso de las personas inmigrantes que se encuentran en una situación administrativa irregular. Aunque la condición de extranjería no afecta a la posibilidad de recibir un curso, existen recursos específicamen- 
te destinados a personas inmigrantes en situación irregularidad. El curso que aquí analizamos y que es ofrecido específicamente para estas personas, si bien es impartido por una entidad religiosa, figura en el catálogo formativo de la administración pública. Esta situación no solo pone de manifiesto el hecho de que las administraciones públicas saben de la existencia de tales recursos, sino también la necesidad de las personas que hacen uso de ellos por falta de recursos públicos. La anterior es una de las características de la distribución de recursos del bienestar en el contexto español. En palabras de Miguel Laparra (2003),

España ha aplicado a rajatabla el principio de subsidiariedad, otorgando un papel preponderante a las instituciones no gubernamentales de base, en lo que se refiere a la prestación y distribución de servicios y prestaciones sociales específicas para los inmigrantes, mientras que los recursos normalizados y genéricos que recibe el conjunto de la ciudadanía son prestados por funcionarios o empleados públicos. (p. 58)

El primer aspecto a señalar es que el acceso diferenciado a los recursos y socioasistenciales reproduce un principio clasificador fundamental en el tratamiento de la inmigración. La distinción entre nacionales y extranjeros, producto del "pensamiento de Estado" (Sayad, 2010), hace que el estatus legal de las personas tenga como efecto el acceso - o no- - a determinados recursos, entre los que se encuentran aquellos que prestan las organizaciones y entidades de formación. Solo quienes tienen permisos de residencia y de trabajo pueden acceder al sistema normalizado de formación para la inserción laboral. Esta clasificación, que no solo distingue sino que, en última instancia, permite o impide accesos y oportunidades, tiene numerosas consecuencias. Como dijimos más arriba, los usuarios reciben un certificado que deja constancia de que realizaron el curso. Ahora bien, para las personas en situación irregular, la acreditación no es oficial y, por lo tanto, no tiene el mismo valor que los que sí lo son, ni tendrá la misma incidencia a la hora de hacerlo valer en un mercado de trabajo que necesariamente será precario (Anthias \& Lazaradis, 2000).

Por lo dicho, los ámbitos de formación para la inserción laboral son un escenario para el despliegue de una de las clasificaciones poblacionales con mayor incidencia en los mundos contemporáneos: la diferencia entre nacionales y extranjeros. Dentro de este último grupo, los inmigrantes son socialmente concebidos como extranjeros y extracomunitarios (Santamaría, 2002), a pesar de que los límites no siempre son claros y que se producen solapamientos y borraduras sociopolíticas. Esta diferencia jerarquizante, introducida por leyes y normativas migratorias, coloca a algunas personas en una situación irregular y en una posición de mayor vulnerabilidad, al limitar su posibilidad de acceso a los lugares menos valorados del mercado de trabajo.

El mayor peso que el trabajo informal tiene en España en relación con otros países de la Unión Europea indica una particular forma de inserción en el mercado internacional del trabajo - antes que a un menor desarrollo- (Gil Araujo \& González-Fernández, 2014; Calavita, 2005). La alta proporción de personas inmigrantes indocumentados es una característica estructural del régimen migratorio español, unos inmigrantes de quienes se ocuparán los cursos de formación impartidos por la iglesia católica y otras organizaciones no gubernamentales.

\section{Aprendiendo los saberes del cuidado: cómo y quiénes}

Como dijimos anteriormente, la distinción entre nacionales e inmigrantes extranjeros tiene varias consecuencias. Entre ellas, destacamos el modo en que se evalúan los saberes de diferentes actores sociales y el lugar que ocupan estos en los contenidos de los cursos y las dinámicas del aula.

En este caso particular, la formación es impartida por mujeres "autóctonas" que traen a las clases su experiencia como trabajadoras domésticas, profesionales del cuidado o amas de casa. Los hombres y mujeres inmigrantes en situación irregular que asisten al curso ponen en juego sus saberes y experiencias previas, desde la posición de desventaja que les otorga su condición de extranjeros irregulares. 
En el desarrollo del curso se tratan los siguientes temas: limpieza, cuidado de niños, planchado, cuidado de ancianos y cocina. La clase de limpieza la imparte una trabajadora doméstica; la del cuidado de ancianos y de niños, estudiantes de un instituto de formación profesional que hacen prácticas como parte de su plan de estudios; las clases de cocina y plancha, las dictan dos amas de casa.

La coordinadora de la formación es quien gestiona el desarrollo del curso: realiza las inscripciones, compra el desayuno para las asistentes al curso, compra los ingredientes para la clase de cocina y lleva la bolsa de trabajo de la entidad. En las entrevistas informales mantenidas con ella y sobre las personas que asisten al curso, dijo: "la gente que viene no tiene mucha cultura", "las sudamericanas son cariñosas", "hay que decirles todo". Estas opiniones y otras fueron expresadas en presencia de las personas a las que aluden y en voz alta. Ello pone de manifiesto, además de los prejuicios que la coordinadora tiene sobre ellas, la distancia y la relación extremadamente desigual entre las participantes y la coordinadora.

La formadora de la clase de limpieza — que es ella misma trabajadora doméstica - realiza en una de sus clases una minuciosa explicación de cómo se debe asear cada parte de la casa, con qué frecuencia, con qué productos y la importancia del orden y la organización para llevar a cabo esta tarea. También ofrece lo que ella misma llama "advertencias", que tienen que ver con el lugar subordinado que les espera a quienes asisten al curso. El siguiente es uno de los ejemplos a los que recurre: si, haciendo el aseo alguna cosa se rompe, es preciso dejarla en la mesa sobre un papel de diario, porque "la señora puede pensar que no se rompió". En este sentido, existe un reconocimiento explícito de la relación desigual que existe entre la empleada doméstica y "la señora". Esta relación es tan desigual que la aprendiz de empleada doméstica, acepta la sospecha de "la señora" sobre su propia honestidad. Este aspecto moral tiene un doble rasero, pues la empleada doméstica acepta que se dude de su honestidad, sin que sea igualmente cuestionada la potencial falta de integridad de "la señora" — a pesar de las lamentables condiciones de empleo en las que se encuentran la mayoría de trabajadoras de esta área-. Argumentamos que, en dichas advertencias, la formadora se identifica con las asistentes al curso que presumiblemente trabajarán, en el futuro, como empleadas domésticas. La relación desigual entre "señora" y "empleada", mediada por el trabajo, se refuerza con otras advertencias, como las siguientes: es deseable que sea la "señora" quien manipule los electrodomésticos; es preferible evitar tener las llaves de la casa; no contar los problemas personales a la señora, pero sí escuchar los de ella — sin darle consejos- Se aconseja que la bata $^{5}$ esté siempre limpia, ya que "la señora" puede notar alguna mancha vieja. Estas consideraciones indican que la posición desde la que se ofrecen tales advertencias es heterónoma, es decir, que depende de la dirección por parte de otra(s) persona(s). En este sentido, hablamos de una desestabilización de las categorías nacional e inmigrante, ya que tanto la formadora como la asistente al curso, en tanto trabajadoras de un hogar que no es el suyo, dependen de la voluntad de 'otra'-la "señora" de la casa en la que trabajan o trabajarán llevando a cabo tareas de cuidado del hogar-.

A lo largo de las clases, emergen maneras distintas de llevar a cabo las tareas de cuidado. Sin embargo, estas no son incorporadas a la dinámica de la clase y tampoco se indaga sobre los conocimientos previos de los participantes. En una ocasión, el tema de la clase fue cómo dar un baño a un niño. Ante la pregunta de la clase, una de las participantes afirmó que, como era mamá, ya sabía muchas cosas, mientras otro de los asistentes aseguró que cambiaba los pañales de su niña. Sin embargo, la clase siguió con el guion preestablecido, fijado en cuadernillos fotocopiados de cada una de las asignaturas, distribuidas al inicio del curso. El siguiente es un ejemplo tomado del diario de campo:

Ana [formadora] coge el muñeco, explica cómo limpiar a una niña de arriba hacia abajo, para no contaminar los genitales. Estos se limpian de aden-

5 Bata es el nombre que se da en España a la prenda holgada y cómoda que se usa para realizar las tareas domésticas. Es sinómimo de guardapolvo o delantal. 
tro hacia afuera por la misma razón. Con el niño es igual. Dice que tienen que quedar secos los pliegues para que no se lastimen. Vanesa [asistente del curso] pregunta si no es mejor lavarlos después de las cacas, Ana dice que eso se enseña con las toallitas (26 de enero de 2010, Clase cuidado de niños).

En ninguno de los casos sobre los modos de higienizar a los niños se recupera o dialoga con los saberes previos de los asistentes al curso, a pesar de que es bastante probable que tuvieran algunos.

En la clase de cocina - cuya profesora es ama de casa-, los asistentes realizan un plato de comida. Esta situación podría ser una oportunidad para que se produzca un intercambio en relación con los alimentos. Sin embargo, los comentarios siempre tienen que ver con los platos tradicionales españoles - paella, cocido de navidad, canelones_, y no se produce ningún intento por introducir otros platos o maneras de prepararlos. Así pues, los conocimientos valorados en el espacio del curso son aquellos ofrecidos por las formadoras; los de los y las participantes, por su parte, son omitidos.

En las clases ofrecidas por las estudiantes-practicantes de formación profesional, los saberes trasmitidos están protocolizados. Las lecciones van desde cómo tender una cama con un anciano acostado y cómo levantar a una persona de una silla hasta cómo introducir una sonda vesical, entre otras. La clase de limpieza, en cambio, está basada en la propia experiencia de la formadora. Así, por ejemplo, ella recomendaba que, cada vez que se cambiara de cuarto, se enchufara la aspiradora en una toma de corriente diferente y evitar así el desgaste en un mismo punto del cable. También recomendaba colocar poco detergente durante la limpieza para que el enjuague fuera más fácil. Por las particularidades de la clase, fue posible que se diera una identificación entre la formadora y las y los asistentes, desestabilizando así la relación jerarquizante entre "nacional" e "inmigrante". Otro elemento desestibilizador de suma importancia son las recomendaciones en relación con las condiciones laborales. La formadora sugiere formas para poner límites a un ámbito fuertemente desregulado y para reconocer cuándo una demanda es excesiva. "Si os dicen tenéis que hacer esto, y esto otro, y esto otro, y tenéis dos horas, no se puede hacer todo, será rápido, una pasada". Hace un gesto con la mano de fregar ${ }^{6}$ rápidamente $(25 \mathrm{de}$ enero de 2010. Clase de limpieza). Además de esta consideración, también se sugerían criterios para cotizar el trabajo y se les recomendaba no cobrar menos de ciertos montos de dinero.

La manera en la que la entidad se lleva la bolsa de empleo marca una relación notoriamente desigual. La coordinadora explica que las personas que han hecho un curso se presentan en la parroquia y se dirigen a ella, manifestándole su disponibilidad para trabajar. El criterio de evaluación para otorgar un empleo depende entonces exclusivamente de la voluntad y decisión de la coordinadora del curso.

Además de establecer relaciones de distanciamiento y proximidad entre las categorías nacional e inmigrante, el curso cumple con la función de ser un espacio de intercambio y una vía de acceso a un trabajo - precario y desregulado pero, para muchos, preferible al desempleo-. Aun cuando no obtengan empleo, las personas que asisten a los cursos formativos pueden recibir el certificado que acredita su paso por una institución de la sociedad, lo que implica un punto a favor a la hora de pedir el permiso de residencia, por el arraigo social y su voluntad de "integración social". Así pues, el curso de formación es valorado por las personas inmigrantes como un lugar que les permite establecer algún tipo de contacto que potencialmente derive en la obtención de un empleo o algún beneficio asociado.

\section{Discusión}

Los modos de aplicar las políticas públicas son complejos y matizados (Shore \& Wright, 1997). Las políticas de control del flujo migratorio y las políticas de integración dirigidas a la inmigración conforman el grueso de las políticas migratorias de una nación y, sin embargo su relación no es de unicidad y coherencia (Yufra, 2014). Como hemos visto aquí, las políticas de integración están atravesadas

6 Forma de referirse a la acción de limpiar, frotar una cosa con un paño o estropajo empapados en agua y generalmente jabón u otro producto de limpieza. 
por políticas de control de los flujos migratorios y, muchas veces, subsanan la situación de vulnerabilidad generada por aquellas otras (Gil Araujo, 2010). La distinción entre nacionales y extranjeros que el Estado impone opera también a nivel de los saberes que portan las personas y de la posición social que ocupan. Así pues, los pares opuestos saber/ ignorancia y quien da/quien recibe el curso dirigen el saber en un solo sentido, quedando los saberes de quienes realizan el curso en posición de privación y desventaja. Al tratarse de saberes prácticos e instrumentales basados en la experiencia, se podría pensar que es pertinente reconocer la legitimidad de los conocimientos previos de las personas que asisten al curso. Sin embargo, lejos de que esto suceda, se reafirma la versión que se imparte en las clases, definiendo así una única manera de hacer las cosas. En este sentido, es posible reconocer que en la formación para la inserción laboral de las personas inmigrantes existe una concepción fuertemente etnocéntrica. En palabras de Natalia Ribas y otros, en dicha formación se expresa una "filosofía asistencialista y asimilacionista, de modo que no aprovechan los conocimientos, los hábitos y las aptitudes que estas mujeres adquirieron en sus sociedades de origen" (Ribas, Alarcón, Gibert, \& Parella, 2000, p. 10).

Los saberes que están en juego son aquellos que señalan cómo hay que cuidar el hogar, saberes del ámbito doméstico, saberes "de mujeres". Suponen la trasmisión a través de las sucesivas generaciones del significado del cuidado, es decir, cómo producir bienestar en las personas que están a cargo. Así las cosas, "el trabajo doméstico, en cuanto trabajo afectivo, implica siempre producir bienestar, habitabilidad, afabilidad y confort. Esto es siempre así, incluso cuando no se demanda explícitamente como una finalidad del empleo" (Gutiérrez-Rodríguez, 2012, p. 130). Son saberes del cuidado que remiten a la afectividad y a la esfera del ámbito privado, a la reproducción de la vida. Dolores Juliano (2004) sostiene que las tareas del cuidado — de la casa, de los niños, de los enfermos-, así como las prestaciones de afecto y servicios sexuales, se consideran tareas propias de mujeres y son valoradas positivamente si se realizan en el marco del ámbito doméstico - aunque en las sociedades capitalistas y patriarcales este sea considerado un ámbito subalterno-. Basta recordar que el trabajo doméstico se rige por un Régimen Especial de los Empleados de Hogar, que se diferencia de los otros tipos de trabajo regidos por el Régimen General de la Seguridad Social española?

Paralelamente, estas mismas tareas, en el mercado de trabajo, asumen un signo valorativo opuesto: son trabajos desregulados, con escasa cobertura legal y social. Por este motivo, consideramos importante destacar las sugerencias y recomendaciones relativas a la necesidad de poner límites y reglas, teniendo en cuenta que se trata de un ámbito desregulado y carente de inspecciones de las condiciones en las que se realiza. Con Peter Spink (2011), podríamos decir que se trata de una trasmisión de saberes que tienen que ver con el "saber arreglárselas". Este tipo de referencia nos permite reconocer la desestabilización de la diferencia jerarquizante entre nacionales y extranjeros-inmigrantes.

La genealogía de la cultura del cuidado en España, ligada a la familia, al hogar y a las mujeres (Vega, 2009), junto con los factores mencionados más arriba, convierten a personas inmigrantes en situación irregular — como aquellas que realizan el

7 A diferencia del Régimen General, el servicio doméstico en España se rige por un Régimen Especial. Esto se debe a que se desarrolla en un ámbito particular: "es precisamente el ámbito de la prestación de servicios, es decir el hogar familiar, el factor determinante de las especialidades que con respecto a la legislación común se prevén en esta norma, ya que ello determina la necesidad de que esta relación se base en la mutua confianza de las partes, equilibrando el respeto a los deberes laborales básicos de los trabajadores con la necesaria flexibilidad que debe concederse a que el empleador y el trabajador determinen las condiciones de prestación de servicios por mutuo acuerdo, no cabiendo tampoco olvidar que en el ámbito familiar en el que se desarrolla el trabajo se proyectan derechos constitucionales, relativos a la intimidad personal y familiar." (BOE 13 agosto 1985, núm. 193). Esto tiene importantes consecuencias, por ejemplo en lo que se refiere a las inspecciones laborales; "La acción de control de cumplimiento de la legislación laboral relativa al contrato de trabajo doméstico, a cargo de la Inspección de Trabajo y Seguridad Social, sólo podrá realizarse salvaguardando los derechos a la inviolabilidad del domicilio y al debido respeto a la intimidad personal y familiar." (BOE 13 agosto 1985, núm. 193). Las modificaciones de este Real Decreto por el Real Decreto 1620/2011 y posteriormente por el Real Decreto 29/2012 mejoran en algunos aspectos de los modos de contratación aunque siguen manteniendo su caractér especial así como el otro aspecto ampliamente cuestionado que se refiere al no reconocimiento al pago del seguro por desempleo para este Régimen Especial. 
curso- en las idóneas para efectuar ciertas "tareas" de escaso valor social, invisibilizadas, con escasa remuneración y cobertura legal. Estas tareas descualificadas, en tanto tales, no son susceptibles de una buena formación. En este sentido, los saberes del cuidado del hogar se encuentran desvalorizados y jerárquicamente diferenciados, en un mercado laboral generizado (Mestre, 2010) y segmentado en función del origen nacional (Catarino \& Oso, 2000) y de la situación administrativa en la que las personas se encuentran. Las tareas asignadas a los hombres son las que representan el hogar hacia el exterior, es decir, se encuentran en un plano socialmente visible, mientras que las tareas que tienen lugar en el interior de los hogares son invisibles - aunque igualmente indispensables, llevadas a cabo por mujeres, para la producción y reproducción de la vida familiar y social- Invisibilidad y necesidad son atributos que comparten las mujeres inmigrantes que llevan a cabo las tareas del cuidado del hogar.

Ese mercado laboral del cuidado, generizado y segmentado, requiere de determinadas disposiciones - explícitamente formuladas en el curso de formación-. Así pues, el curso deviene transmisor de las actitudes que se esperan de una posición social subordinada de la "empleada", en relación con la "señora". No obstante, en ese proceso de trasmisión es posible encontrar relaciones que rompen la distancia entre quienes dictan el curso y las personas inmigrantes que lo reciben, en tanto las primeras realizan —o han realizado-aquellas labores para las que las asistentes al curso están siendo preparadas. Frente a la "señora", tanto la formadora como la persona inmigrante que recibe la formación son "empleadas". A este momento de identificación entre ellas le sucederán momentos de distanciamiento o diferenciación, debido a que las jerarquías establecidas en función del origen nacional reinscriben los borramientos sociales.

Durante el desarrollo del curso fue posible reconocer, simultáneamente, la complejidad y el esfuerzo que supone llevar a cabo las labores del cuidado y su escasa posibilidad de remuneración y reconocimiento, lo que incide en la persistencia de dicha desvalorización social. En este contexto, las desigualdades de género no son cuestionadas y mucho menos superadas: son, más bien transferidas de unas mujeres a otras, siendo las personas inmigrantes —en su mayoría mujeres - quienes realizan mayoritariamente los trabajos domésticos y de cuidado (Montenegro \& Montenegro, 2013). El análisis del curso pone de manifiesto la necesidad de recuperar el carácter vertebrador del cuidado para la reproducción de la vida (Carrasco, 2013) y la de reflexionar sobre las soluciones hasta ahora encontradas para asumir dicho carácter.

Una de las consecuencias de la persistencia de las distinciones jerarquizantes entre nacional y extranjero, o entre autóctono e inmigrante. es la segmentación de derechos sociales. Esta segmentación favorece la idea de que existen sujetos con derechos menguados o, incluso, sin derechos.

\section{Conclusión}

Con el fin de recapitular, diremos que nuestra intención ha sido contribuir a la elucidación de las dinámicas que se producen en ciertos espacios y formas de intervención social de carácter formativo. Nos encontramos con espacios complejos que requirieron cuestionar la mirada unilateral de lo que se propone como 'ayuda' o 'sostén' para un grupo desfavorecido. Dicha elucidación buscó, en su versión crítica de las prácticas de intervención social, explorar las — limitadas_ posibilidades emancipadoras contenidas en tales procesos; unas prácticas que, necesariamente, han de ser reflexivas — como también deben serlo los procesos de investigación social-.

En el espacio de formación para la inserción laboral que analizamos, se refuerza la jerarquía de los saberes que se transmiten en estos dispositivos. La validez de tales saberes se determina en función de las posiciones sociales ocupadas por quienes los detentan, pasando así desapercibidos o siendo desechados valiosos aportes y múltiples maneras de hacer y abordar los cuidados del hogar. Asimismo, la forma que asume el curso omite otras posibles maneras de intervención y acción social más cooperativas — $\mathrm{O}$, al menos, no tan desiguales- Cuando se produce la identificación como trabajadoras cui- 
dadoras del hogar entre nacionales y extranjeras. En este sentido, creemos que es posible tejer alianzas cuando se desarticula la oposición nacional/ extranjero - por ejemplo, cuando se llevan a cabo acciones sociales en términos de reivindicaciones de derechos laborales o, más modestamente, cuando se transmiten estrategias para poner límites a los posibles abusos cotidianos e invisibilizados-. Este representa un terreno fecundo para la exploración de posibilidades que alienten formas de relaciones sociales menos desiguales.

Asimismo, el análisis que hemos llevado a cabo refleja la amenaza que supone el proceso de taxonomizar y clasificar la población entre quienes tienen acceso a determinados recursos públicos y quienes no. Lo anterior, porque se produce una fragmentación de los derechos sociales que, si bien atañen (o no) a las personas extranjeras en función de su situación jurídica, consolida la idea de que existen sujetos sin derechos —o mermados de ellos- en el seno de las propias sociedades democráticas, lo cual incide en su desarrollo y modo de funcionamiento. El contexto de crisis económica en el que se desarrolló el trabajo alerta sobre las consecuencias a largo plazo de la naturalización de dicha fragmentación de derechos sociales.

Los desafíos que emergen del presente recorrido, como hemos visto, son muchos. Para terminar, y en lo que nos toca más directamente, es importante mencionar la necesidad de dar cabida, en la investigación social, a la valorización de las tareas prácticas y manuales, reconociendo su rol indispensable en la reproducción de la vida. También destacamos la importancia de reconocer la diversidad de modos de cuidar y su necesario ejercicio en la vida en sociedad.

\section{Referencias}

Anderson, B. (2000). Doing the dirty work? The global politics of domestic labor. Londres: Zed Publishers.

Anthias, F., \& Lazaradis, G. (Eds.) (2000). Gender and Migration in Southern Europe. New York: Berg.

Berteaux, D. (2005). Los relatos de vida: perspectiva etnosociológica. Barcelona: Bellaterra.
Bourdieu, P. (2008). El sentido práctico. Madrid: Siglo XXI.

Brah, A. (2004). Diferencia, diversidad y diferenciación. En Otras inapropiables. Feminismos desde las fronteras. Madrid: Traficantes de sueños. 107-137.

Carrasco Bengoa, C. (2013). El cuidado como eje vertebrador de una nueva economía. Cuadernos de relaciones laborales, 31(1), 39-56.

Catarino, C., \& Oso, L. (2000). La inmigración femenina en Madrid y Lisboa. Hacia una etnización del servicio doméstico y de las empresas de limpieza. Papers, 60, 183-207.

Calavita, K. (2005). Immigrants at the margins. Law, race and exclusion in southern Europe. Cambridge: Cambridge University Press.

Hammersley, M., \& Atkinson, P. (1994). Etnografía. Métodos de investigación. Barcelona: Paidós.

Ferrera, M. (1996). The «southern model» of welfare in social Europe. Journal of European Social Policy, 6(1), 17-37.

Gil Araujo, S. (2010). Las argucias de la integración. Politicas migratorias, construcción nacional y cuestión social. Madrid: Iepala.

Gil Araujo, S., \& González-Fernández, T. (2014). International migration, public policies and domestic work Latin American migrant women in the Spanish domestic work sector. Women's Studies International Forum, 46, 13-23.

Gutiérrez-Rodríguez, E. (2013). Trabajo domésticotrabajo afectivo: sobre heteronormatividad y la colonialidad del trabajo en el contexto de las políticas migratorias de la UE. Revista de Estudios Sociales, 45, 123-134.

Foro para la Integración Social de los Inmigrantes (2011). Informe sobre la situación de la integración social de los inmigrantes y refugiados en 2011. Secretaría de Inmigración y Emigración. Ministerio de Trabajo y asuntos sociales. Madrid.

Juliano, D. (2004). Excluidas y marginales: una aproximación antropológica. Madrid: Cátedra.

Laparra, M. (Ed.). (2003). Extranjeros en el purgatorio: integración social de los inmigrantes en el espacio local. Barcelona: Bellaterra.

Mestre, R. (2010). Mujeres, nueva ciudadanía y trabajo: ¿de qué mujeres hablamos? En Freixanet Mateo, M. (Coord.), Dones migrades treballadores. Anàlisi i 
experiències locals contra la desigualtat (pp. 75-107). Barcelona: ICPS.

Montenegro, M. (2001). Conocimientos, agentes y articulaciones: una mirada situada a la intervención social (Tesis doctoral). Facultad de Psicología, Universitat Autònomae Barcelona.

Montenegro, M., Galaz, C., Yufra, L. y Montenegro, K. (2011). Dinámicas de subjetivación y diferenciación en servicios sociales para mujeres inmigradas en la ciudad de Barcelona. Athenea Digital, 11(2), 113-132.

Montenegro, K., \& Montenegro M. (2013). Governmentality in service provision for migrated women in Spain. Social and Personality Psychology Compass, 7(6), 331-342.

Moreno, L. (2002). Bienestar mediterráneo y "supermujeres". Revista Española de Sociología, 2, 41-57.

Parella Rubio, S. (2000). El trasvase de desigualdades de clase y etnia entre mujeres: los servicios de proximidad. Papers, 60, 275-289.

Pedone, C. (2002). Las representaciones sociales en torno a la inmigración ecuatoriana a España. Íconos, 14, 56-66.

Ribas, N., Alarcón, A., Gibert, F., \& Parella, S. (2000). Políticas de formación para mujeres inmigrantes. El caso de la ciudad de Barcelona. Papers, 60, 365-379.

Sassen, S. (2003). Contrageografías de la globalización: género y ciudadanía en los circuitos transfronterizos. Madrid: Traficantes de Sueños.

Sanahuja i Velez, R. (2010). El impacto social de la crisis. El papel del mundo local en la integración de la inmigración. Estudio de caso de Barcelona. En E. Aja, J. Arango, \& J. Oliver (Dirs.), Inmigración y crisis económica. Impactos actuales y perspectivas de futuro. Anuario de la inmigración en España 2010. Bellaterra: Cidob.

Santamaría, E. (2002). La incógnita del extraño. Una aproximación a la significación sociológica de la 'inmigración no comunitaria'. Barcelona: Anthropos.

Sayad, A. (2010). La doble ausencia: de las ilusiones del emigrado a los padecimientos del inmigrado. Barcelona: Anthropos.

Shore, C., \& Wright, S. (Eds.) (1997). Anthropology of policy. Critical perspectives on governance and power. London: Routledge.

Spink, P. (2011). ¿Qué pasó con el trabajo? De la centralidad de los zapatos, barcos y lacre a los problemas planteados por los cerdos volando. Athenea Digital, 11(3), 3-23.

Taylor, S., \& Bodgan, R. (1986). Introducción a los métodos cualitativos de investigación: la búsqueda de significados. Buenos Aires: Paidós.

Vargas-Monroy, L., \& Pujal, M. (2013). Gubernamentalidad, dispositivos de género, raza y trabajo: la conducción de la conducta de las mujeres trabajadoras. Universitas Psychologica, 12(4), 1255-1267.

Vega Solís, C. (2009). Culturas del cuidado en transición: espacios, sujetos e imaginarios en una sociedad de migración. Barcelona: UOC.

Velasco, H., \& Díaz de Rada, Á. (1997). La lógica de la investigación etnográfica: un modelo de trabajo para etnógrafos de la escuela. Madrid: Trotta.

Yufra, L. C. (2014). Tópicos, supuestos e interrogantes en el estudio de y la intervención social con las migraciones internacionales: reflexiones desde España. REMHU: Revista Interdisciplinar da Mobilidade Humana, 22(42), 247-261. 
